\title{
Glucocorticoids Increase Fracture Risk and Fracture Prevalence Independently from Bone Mineral Density and Clinical Risk Factors: Results from the Gliwice Osteoporosis (GO) Study
}

Authors

Wojciech Pluskiewicz¹, Piotr Adamczyk², Bogna Drozdzowska³

Affiliations

1 Department and Clinic of Internal Diseases, Diabetology, and Nephrology, Faculty of Medical Sciences in Zabrze, Medical University of Silesia, Katowice, Poland

2 Department of Paediatrcs, Faculty of Medical Sciences in Katowice, Medical University of Silesia, Katowice, Poland

3 Department of Pathomorfology, Faculty of Medical Sciences in Zabrze, Medical University of Silesia, Katowice, Poland

Key words

fracture prevalence, fracture risk, fracture probability, glucocorticoids

received 24.08.2021

accepted after revision $\quad 15.11 .2021$

Bibliography

Horm Metab Res 2022; 54: 20-24

DOI 10.1055/a-1700-5007

ISSN 0018-5043

(C) 2022. Thieme. All rights reserved.

Georg Thieme Verlag KG, Rüdigerstraße 14,

70469 Stuttgart, Germany

Correspondence

Wojciech Pluskiewicz

Department and Clinic of Internal Diseases

Diabetology and Nephrology

Metabolic Bone Diseases Unit

Faculty of Medical Sciences in Zabrze

Medical University of Silesia in Katowice

3-Maja 13/15 street

41-800

Zabrze

Poland

Tel.: + 48/323/704 389

osteolesna@poczta.onet.pl

\section{ABSTRACT}

The aim of the study was to establish the influence of glucocorticoids (GC) on fracture risk, probability, and prevalence. A set of 1548 postmenopausal women were divided into study group - treated with GC $(n=114$, age $66.48 \pm 7.6$ years $)$ and controls $(n=1434$, age $66.46 \pm 6.83$ years). Data on clinical risk factors for osteoporosis and fractures were collected. Hip bone densitometry was performed using a device Prodigy (GE, USA). Fracture probability was established by FRAX, and fracture risk by Garvan algorithm and POL-RISK. Fracture risk and fracture probability were significantly greater for GC-treated women in comparison to controls. In the study group, there were 24,3 , 24 , and 6 fractures noted at spine, hip, forearm, and arm, respectively. The respective numbers of fractures reported in controls at those skeletal sites were: 186, 23, 240, and 25 . The use of GCs increased significantly prevalence of all major, spine and arm fractures. Also the number of all fractures was affected by GC use. Following factors significantly increased fracture probability: age (OR 1.04 per each year; $95 \% \mathrm{Cl}: 1.03-1.06)$, GC use (OR 1.54; $95 \%$ Cl: 1.03-2.31), falls (OR 2.09; $95 \% \mathrm{Cl}$ : $1.60-2.73$ ), and FN T-score (OR 0.62 per each unit; $95 \% \mathrm{Cl}$ : $0.54-0.71)$. In conclusion, in patients treated with GCs the fracture risk, probability, and prevalence were increased. This effect was evident regardless of whether GC therapy is included in the algorithm as a risk factor (FRAX, POL-RISK) or not taken into consideration (Garvan nomogram).

\section{Introduction}

Osteoporosis is a common disease of modern civilization. In addition to involutional types of osteoporosis, secondary causes of bone loss play an increasing role. Glucocorticoids (GC) therapy is the most common cause of secondary osteoporosis and the leading iatrogenic cause of the disease [1]. GC therapy shows a beneficial influence in various medical conditions, mainly those of inflammatory or immunological pathomechanism. However, GC use may 
lead to several side effects including diabetes, hypertension, cataract, skin lesions, or obesity. One of the most important negative consequence of GC use is bone loss and osteoporotic fractures. GC inhibits bone formation accompanied by an early but transient increase in bone resorption [2]. In the course of GC therapy, the most important consequence associated with the impact of this therapy on the skeletal status is the increased risk of fractures. Increased fracture prevalence due to GC therapy was described in several studies [3-10]. Vertebral fractures are the most common as this skeletal site is rich in trabecular bone, especially sensitive to negative influence of GCs. It is well documented that GCs cause a decline in bone mineral density (BMD) but increased bone fragility in subjects treated with GCs is even higher than one could expected based on changes in BMD. This may indicate that not only impaired bone quantity should be considered as the risk factor for fractures. The increased fracture risk may result also from deterioration of bone structure as was stated in CaMos Study [5]. Therefore, fracture risk related to GC use cannot be directly attributed to bone mass changes. FRAX, a world-spread tool designed to establish fracture probability, includes GC among several other clinical risk factors [11]. Also another algorithm called POL-RISK (www.fracture-risk.pl) comprises GC as a risk factor [12]. The fracture probability assessed with FRAX in patients receiving GCs has been presented in two recent studies $[13,14]$.

The aim of the current cross-sectional study was to establish the influence of GC therapy on fracture risk and fractures prevalence. To our knowledge current study in the first one showing the data on fracture risk established not only by FRAX, but also by Garvan nomogram and POL-RISK algorithm in a female population on GC treatment.

\section{Subjects and Methods}

The presented study is a continuation of analysis in a cohort of women from 'Gliwice Osteoporosis Study' (GO Study). The study group was described in detail in our previously published papers [15-17]. Briefly, the study group was gathered in one outpatient osteoporotic clinic in Gliwice, South of Poland. The overall aim of the GO Study was to present the results of bone health in a female cohort that may be interesting and helpful for practitioners. A huge amount of details possibly affecting bone health have been gathered in the GO Study database. In previously published papers data regarding i.a. height loss $[15,17]$ and dietary calcium intake [16] were utilized.

In the current study, data for 1548 women are presented; among them 114 subjects had reported GC use. For enrolment purposes, the exposure to GC use was defined in the same way as in FRAX calculator - subjects currently exposed to oral GCs or those who have been exposed to oral GCs for more than 3 months at a dose of prednisolone of $5 \mathrm{mg}$ daily or more (or equivalent doses of other GCs) were classified as GC group. For former GC users, the period from end of therapy to the current evaluation was not limited. The control group consists of 1434 subjects. This group was recruited from the whole GO Study cohort $(n=1735)$. All GC-treated women who met the inclusion criteria were enrolled. Control subjects were selected to achieve age-matched and body sizematched comparative group. Therefore, controls did not differ from study group in regard to mean age, body weight, height and BMI. Clinical characteristics for the whole cohort and subgroups is presented in $>$ Table 1.

Subjects included in the study and controls were not treated with antiresorptive drugs and did not receive any medications other than GCs with a possible negative effect on bone health.

Clinical data potentially related to GC use were gathered. Following parameters were established: height loss ( $\mathrm{HL}$, defined as the difference between current height measured by stadiometer SECA, Germany and maximal historical height), bone densitometry result (T-score for femoral neck, FN), prior osteoporotic fractures, fracture probability calculated according to FRAX [11], and fracture risk Garvan [18, 19], and POL-RISK [12] algorithms. Bone mineral density was established using densitometer device Prodigy (GE, USA). Hip was measured at non-dominant side. All measurements were performed by one experienced operator. Based on repeated measurements the precision (CV\%) of DXA measurements at $\mathrm{FN}$ was established at $1.6 \%$.

Statistical analysis was performed, using the Statistica software (StatSoft, Tulsa, OK, USA). The mean values and standard deviations were used for descriptive statistics of continuous variables. The normality of data distribution was verified by the Shapiro-Wilk test. Absolute values and percentage were given for qualitative variables. The Student's $t$-test for independent samples or the MannWhitney U-test was applied for comparative analyses of continuous variables, depending on data distribution. Comparisons of qualitative features frequency were performed by the chi-square test. In order to evaluate the independent influence of the analyzed factors on the fracture prevalence, logistic regression was used. The significance of results in all the statistical analyses was assumed at $p<0.05$.

\section{Results}

Mean values of T-score for $\mathrm{FN}$ in the study group and controls were $-1.98 \pm 0.86$ and $-1.67 \pm 0.90$, respectively, and did not differ significantly. Mean $\mathrm{HL}$ in the study and control group were $4.51 \pm 4.04$ and $3.54 \pm 2.84 \mathrm{~cm}$, respectively, and differed significantly $(p<0.0001)$. The results of fracture risk and fracture probability are presented in $>$ Table 2 . The 10 -year facture probability established by FRAX (for major and hip fractures), as well as 5-year and 10 -year fracture risk by Garvan (for any or hip fractures) and

- Table 1 Clinical characteristics for the whole cohort, as well as for study and control groups.

\begin{tabular}{|l|c|c|c|}
\hline Parameter & $\begin{array}{l}\text { Whole cohort } \\
\mathbf{n = 1 5 4 8}\end{array}$ & $\begin{array}{l}\text { Study group } \\
\mathbf{n = 1 1 4}\end{array}$ & $\begin{array}{l}\text { Control group } \\
\mathbf{n = 1 4 3 4}\end{array}$ \\
\hline Age (years) & $66.5 \pm 6.9$ & $66.5 \pm 7.6$ & $66.5 \pm 6.8$ \\
\hline Weight $(\mathrm{kg})$ & $69.5 \pm 13.2$ & $68.8 \pm 14.0$ & $69.6 \pm 13.1$ \\
\hline Height $(\mathrm{cm})$ & $157.6 \pm 6.1$ & $157.3 \pm 6.2$ & $157.6 \pm 6.1$ \\
\hline BMI $\left(\mathrm{kg} / \mathrm{m}^{2}\right)$ & $28.0 \pm 5.1$ & $27.8 \pm 5.6$ & $28.0 \pm 5.1$ \\
\hline
\end{tabular}

No significant differences between study and control groups. 
- Table 2 The results for fracture risk/probability in GC-treated women and controls.

\begin{tabular}{|c|c|c|c|}
\hline Fracture risk & $\begin{array}{l}\text { Study group } \\
n=114\end{array}$ & $\begin{array}{l}\text { Control group } \\
n=1434\end{array}$ & p-Value \\
\hline $\begin{array}{l}\text { FRAX - major } \\
\text { fractures }\end{array}$ & $13.3 \pm 8.4$ & $6.7 \pm 4.4$ & $<0.0001$ \\
\hline $\begin{array}{l}\text { FRAX - hip } \\
\text { fractures }\end{array}$ & $5.5 \pm 6.5$ & $2.2 \pm 3.6$ & $<0.0001$ \\
\hline $\begin{array}{l}\text { Garvan } \\
\text { - } 5 \text {-year any } \\
\text { fractures }\end{array}$ & $16.9 \pm 14.3$ & $12.8 \pm 11.5$ & $<0.001$ \\
\hline $\begin{array}{l}\text { Garvan } \\
-10 \text {-year any } \\
\text { fractures }\end{array}$ & $30.4 \pm 21.2$ & $23.8 \pm 17.5$ & $<0.001$ \\
\hline $\begin{array}{l}\text { Garvan } \\
\text { - 5-year hip } \\
\text { fractures }\end{array}$ & $8.3 \pm 12.8$ & $5.1 \pm 9.4$ & $<0.001$ \\
\hline $\begin{array}{l}\text { Garvan } \\
\text { - 10-year hip } \\
\text { fractures }\end{array}$ & $14.4 \pm 18.9$ & $9.0 \pm 14.0$ & $<0.001$ \\
\hline $\begin{array}{l}\text { POL-RISK } \\
\text { - 5-year any } \\
\text { fractures }\end{array}$ & $27.4 \pm 16.3$ & $12.5 \pm 7.7$ & $<0.0001$ \\
\hline
\end{tabular}

5-year fracture risk according to POL-RISK tool for any fractures, were significantly higher in the study group in comparison to controls. We also analyzed data for prevalence of separate clinical risk factors for fractures (smoking, secondary osteoporosis, falls, rheumatoid arthritis, hip fractures in parents) and those results are presented in $>$ Table 3 . Only the frequency of rheumatoid arthritis was significantly higher in GC-treated women in comparison to controls; the prevalence of other risk factors did not differ between subgroups in chi-square test.

In the study group, there were 56 subjects who reported at least one fracture (49.1\%) and in control group fractures were observed in 510 women (35.6\%). Taking into account major osteoporotic fractures, in the study group there were $24,3,24$, and 6 fractures noted at spine, hip, forearm and arm, respectively. The respective numbers of fractures reported in controls at those skeletal sites were: $186,23,240$, and 25.

As shown by Chi-square test the use of GCs increased significantly prevalence of all major osteoporotic fractures, spine fractures and arm fractures. Also the number of all fractures was affected by GC use. The frequency of mentioned above fractures in GC-treated women and in controls is presented in $>$ Fig. 1. The prevalence of forearm fractures did not differ significantly between study and control groups ( 21.0 vs. $16.7 \%$, respectively). Comparison of hip fractures prevalence was not informative due to small number of hip fractures in the study cohort.

Finally, logistic regression analysis was performed in order to establish which factors independently increase fracture prevalence in the study group. In the initial model age, BMI, height, GC use, rheumatoid arthritis, smoking, hip fracture in parents, secondary osteoporosis, falls and T-score value were taken into consideration.
- Table 3 The prevalence of clinical risk factors for fractures in GC-treated women and controls.

\begin{tabular}{|l|l|l|l|}
\hline Fracture risk factor & $\begin{array}{l}\text { Study } \\
\text { group } \\
\mathbf{n = 1 1 4}\end{array}$ & $\begin{array}{l}\text { Control } \\
\text { group } \\
\mathbf{n = 1 4 3 4}\end{array}$ & p-Value \\
\hline Smoking & $14.9 \%$ & $12.8 \%$ & NS \\
\hline Rheumatoid arthritis & $12.3 \%$ & $0.7 \%$ & $<0.0001$ \\
\hline Falls & $24.6 \%$ & $19.0 \%$ & NS \\
\hline Hip fractures in parents & $8.8 \%$ & $5.2 \%$ & NS \\
\hline Secondary osteoporosis & $4.4 \%$ & $5.5 \%$ & NS \\
\hline
\end{tabular}

In the final model following factors were identified as significantly increasing fracture probability: age (OR 1.04 per each year; $95 \%$ Cl: 1.03-1.06), GC use (OR 1.54; $95 \%$ Cl: 1.03-2.31), falls (OR 2.09; $95 \% \mathrm{Cl}: 1.60-2.73$ ) and FN T-score (OR 0.62 per each unit; $95 \% \mathrm{Cl}$ : $0.54-0.71)$.

\section{Discussion}

To the best of our knowledge the presented study is the first one showing the data on fracture risk established by both Garvan and POL-RISK calculators together with FRAX probability estimation in a female population on GC treatment. The most significant finding is the observation that for all methods expressing fracture risk or probability subjects with prior GC use present an increased value of estimated parameter. More importantly, such result was even noted for the Garvan method, although the GC use is not a risk factor included in the Garvan model.

One might suspect that the increased fracture risk was found in subjects with GC use due to more frequent presence of other risk factors for fracture which are correlated with GC treatment. Beside higher frequency of rheumatoid arthritis (what is obvious because GC are often prescribed in those patients) the frequency for all other clinical risk factor did not differ between subgroups. Moreover, also bone status expressed by the value of T-score for FN did not differ. Summarizing this part of discussion we emphasize that GC use is an independent, important factor increasing significantly fracture risk. This observation is confirmed by analysis of fracture prevalence, and generally GC patients had significantly more fractures than controls.

An interesting observation of the influence of $G C$ use was shown in patients with rheumatoid arthritis [14]. The authors noted significantly higher risk for fractures, both for major and hip fractures. Respective results according to FRAX in patients and controls were: 14 versus 8 and 4.4 versus 2.0. FRAX values for major fractures were similar to the same results found in our current study, namely approximately 13 in GC treated and 7 in GC naïve women ( $\bullet$ Table 2.). Also adequate data for hip fractures were comparable, we noted 5 and 2 and cited authors 4.4 and 2.2 for treated and untreated, respectively. The values of fracture prevalence were comparable with our data but the mean age was lower (57-58 vs. 66 years in current study). In a position paper focused on the impact of GC on FRAX 


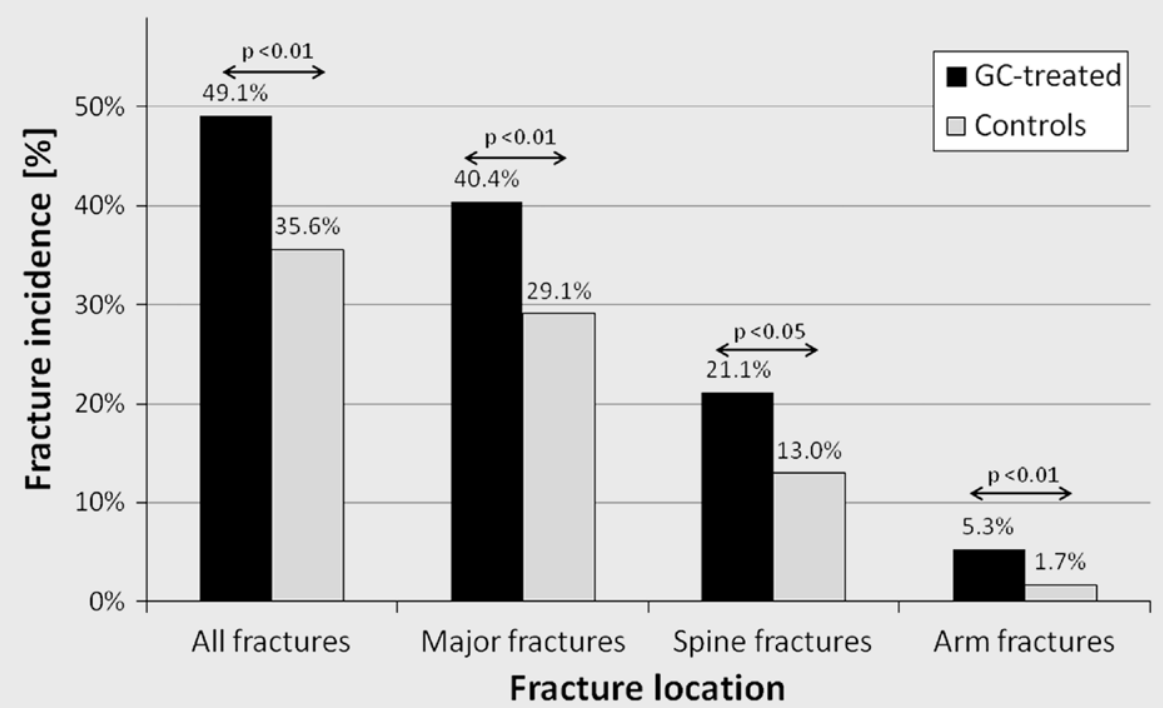

- Fig. 1 Fracture prevalence [\%] for all fractures, major fractures, spine and arm fractures in GC-treated women and in controls.

results the increase of fracture risk varied from $10-25 \%$ in various age subgroups and fracture site [13]. One should also note that FN BMD did not differ between patients and controls, which was also noted in the current study. GCs affect not only bone mineralization but also bone strength and structure, which clearly indicates that bone densitometry is not the optimal tool for the assessment of bone status is those patients. Probably, quantitative ultrasound is more appropriate method because it reveals both quantitative and qualitative features of bone tissue [20,21]. In mentioned studies in female and male patients with bronchial asthma taking GC skeletal status was assessed using quantitative ultrasound at the calcaneus, and this method was found useful to follow skeletal changes during GC therapy. However, the availability of quantitative ultrasound devices is not very wide. Therefore, probably the most recommended way for fracture risk assessment are diagnostic tools used in current study (FRAX, Garvan, POL-RISK). We consider that this observation is the most important finding for practitioners.

Fractures are the most important event in osteoporotic populations. Therefore, we consider that the observations of increased fracture prevalence among patients taking GC are also a significant clinical finding. In several other studies such point was a matter of interest [3-10] and general view is comparable in many studies.

Our study has several limitations. The study was not based on a prospective observation. The study group was not an epidemiological, population representative sample. Only women were included. However, a size of study cohort, wide range of risk factors collected and the use of three online available methods for fracture risk assessment allowed for presentation of valuable clinical data.

\section{Conclusion}

In postmenopausal women treated with GCs, the fracture risk and probability calculated by online available tools (FRAX, Garvan, POL-
RISK) are increased. This effect was evident regardless of whether GC therapy is included in the algorithm as a risk factor (FRAX, POLRISK) or not taken into consideration (Garvan nomogram). The negative influence of GCs on skeletal status was not only noticeable in the risk estimation, but also confirmed by the observed higher prevalence of fractures in GC-treated women.

\section{Author Contributions}

Pluskiewicz Wojciech - study design, collecting data, writing the first draft of the manuscript; Adamczyk Piotr - statistical analysis of collected data, writing some parts of the manuscript, preparing tables and figure; Drozdzowska Bogna - writing some parts of the manuscript, review of the manuscript.

\section{Conflict of Interest}

The authors declare that they have no conflict of interest.

\section{References}

[1] Adler RA, Curtis JR, Saag K et al. Glucocorticoid-induced osteoporosis. 3rd ed.San Diego: Elsevier-Academic Press; 2008

[2] Compston J. Glucocorticoid-induced osteoporosis: an update. Endocrine 2018; 61: 7-16

[3] Mazzantini M, Torre C, Miccoli M et al. Adverse events during long term low-dose glucocorticoid treatment of polymyalgia rheumatica: a retrospective study. J Rheumatol 2012; 39: 552-557

[4] Majumdar SR, Morin SN, Lix LM et al. Influence of recency and duration of glucocorticoid use on bone mineral density and risk of fractures: population-based cohort study. Osteoporos Int 2013; 24: 2493-2498 
[5] Ioannidis G, Pallan S, Papaionannou A et al. CaMos research group. Glucocorticoids predict 10 -year fragility fracture risk in a population-based ambulatory cohort of men and women: Canadian multicentre osteoporosis study (CaMos). Arch Osteoporos 2014; 8: 169

[6] Amiche MA, Albaum JM, Tadrous M et al. Fracture risk in oral glucorticoid users: a Bayesian meta-regression leveraging control arms of osteoporosis clinical trials. Osteoporos Int 2016; 27: 1709-1718

[7] Balasubramanian A, Wade SW, Adler RA et al. Glucocorticoid exposure and fracture risk in a cohort of US patients with selected conditions. J Bone Miner Res 2018; 33: 1881-1888

[8] Robinson DE, van Staa TP, Dennison EM et al. The limitations of using simple definitions of glucocorticoid exposure to predict fracture risk: a cohort study. Bone 2018; 117: 83-90

[9] Chang YP, Lai CH, Lin CY et al. Mortality and vertebral fracture risk associated with long-term oral steroid use in patients with chronic obstructive pulmonary disease: a systematic review and meta-analysis. Chron Respir Dis 2019; 16: 1-7

[10] Che H, Breuil V, Cortet B et al. Vertebral fractures cascade: potential causes and risk factors. Osteoporos Int 2019; 30: 555-563

[11] Kanis JA, Johnell O, Oden A et al. FRAX ${ }^{\mathrm{TM}}$ and the assessment of fracture probability in men and women from the UK. Osteoporos Int 2008; 19: 385-397

[12] Adamczyk P, Werner A, Bach M et al. Risk factors for fractures identified in the algorithm developed in 5-year follow-up of postmenopausal women from RAC-OST-POL study. J Clin Densitom 2018; 21: 213-219

[13] Leib ES, Saag KG, Adachi JD et al. Official positions for FRAX(®) clinical regarding glucocorticoids: the impact of the use of glucocorticoids on the estimate by $\operatorname{FRAX}(\circledR)$ of the 10 year risk of fracture from joint official positions development conference of the international society for clinical densitometry and international osteoporosis foundation on FRAX(®). J Clin Densitom 2011; 14: 212-219
[14] Cheng TT, Lai HM, Yu SF et al. The impact of low-dose glucocorticoids on disease activity, bone mineral density, fragility fractures, and 10-year probability of fractures in patients with rheumatoid arthritis. Investig Med 2018; 66: 1004-1007

[15] Pluskiewicz W, Adamczyk P, Drozdzowska B. The significance of height loss in postmenopausal women. The results from GO Study. Int J Clin Pract 2021; 75: e14009

[16] Pluskiewicz W, Adamczyk P, Drozdzowska B. Low dietary calcium intake does not modify fracture risk but increases falls frequency: the results of GO Study. Endokrynol Pol 2021; 72: 198-201. doi:10.5603/ EP.a2021.0021

[17] Pluskiewicz W, Adamczyk P, Drozdzowska B. Height loss in postmenopausal women-do we need more for fracture risk assessment? Results from the GO Study. Osteoporos Int 2021; 32: 2043-2049

[18] Nguyen ND, Frost SA, Center JR et al. Development of a nomogram for individualizing hip fracture risk in men and women. Osteoporos Int 2007; 18: 1109-1117

[19] Nguyen ND, Frost SA, Center JR et al. Development of prognostic nomograms for individualizing 5-year and 10-year fracture risks. Osteoporos Int 2008; 19: 1431-1434

[20] Drozdzowska B. Skeletal status assessed by quantitative ultrasound at the calcaneus in females with bronchial asthma on prolonged corticosteroid therapy. Maturitas 2005; 51: 386-392

[21] Drozdzowska B. Quantitative ultrasound at the calcaneus in corticosteroids-treated male patients with bronchial asthma. Ultrasound in Med and Biol 2004; 30: 1057-1061 